\title{
TLE3 Gene
}

National Cancer Institute

\section{Source}

National Cancer Institute. TLE3 Gene. NCI Thesaurus. Code C99617.

This gene is involved in transcriptional repression. 